\title{
Technologien zur Nutzung von Wertstoff- und Energiepotentialen in LD-Konverterschlacken
}

\author{
Alexandra Holzer, Valentin Mally, Christoph Ponak, Stefan Windisch und Harald Raupenstrauch \\ Lehrstuhl für Thermoprozesstechnik, Montanuniversität Leoben, Leoben, Österreich \\ Eingegangen 30. April 2020; angenommen 11. Mai 2020; online publiziert 25. Mai 2020
}

Zusammenfassung: Zwei Drittel der weltweiten Rohstahlerzeugung erfolgt über die die Hochofen-Konverter-Route. Im Sauerstoffkonverter werden neben Kohlenstoff, Silizium und Phosphor auch Wertmetalle wie Chrom, Mangan und Eisen aus dem Roheisen entfernt. In diesem Artikel wird ein Verfahren beschrieben, das die Möglichkeit eröffnen soll, diese in den Produktkreislauf rückzuführen. Die Aufgabenstellung dabei ist die Vermeidung der Bildung einer Verbindung aus Eisen und Phosphor zu Phosphiden, da diese bei Wiedereinsatz der Recyclingprodukte im Hüttenwerk zu einer Phosphoranreicherung im Rohstahl führen. In einem neuartigen Reaktorkonzept, welches am Lehrstuhl für Thermoprozesstechnik der Montanuniversität Leoben entwickelt wurde, ist die vollständige Rückgewinnung der Metalle mit gleichzeitiger Entfernung des Phosphors über die Gasphase möglich. Dies basiert auf dem Prinzip des induktiven Wärmeeintrags über ein Grafitbett, welches eine große Oberfläche für Reduktionsreaktionen bietet. In einer diskontinuierlichen Vorversuchsreihe wurden sowohl eine optimierte Mischung aus LD-Schlacke und Schlackenbildnern wie auch die optimale Betriebstemperatur ermittelt. Folglich kann das Einsatzgemisch erfolgreich im kontinuierlichen Betrieb getestet werden. Die Ergebnisse zeigen, dass die Schlacke nach der Behandlung nahezu vollständig frei von Eisen, Chrom und Phosphor ist.

Schlüsselwörter: Stahlwerksschlacke,

Phosphorrückgewinnung, Schlackenreduktion,

Metallrückgewinnung

Technologies for the Utilization of Valuable Material and Energy Potentials in Basic Oxygen Furnace Slags

Abstract: Two thirds of global crude steel production is based on the blast furnace-basic oxygen furnace route (BF-

$\overline{\text { Dipl.-Ing. A. Holzer }(\bowtie)}$

Lehrstuhl für Thermoprozesstechnik,

Montanuniversität Leoben,

Franz Josef-Straße 18,

8700 Leoben, Österreich

alexandra.holzer@unileoben.ac.at
BOF route). In addition to carbon, silicon, and phosphorus, valuable metals, such as chromium, manganese, and iron, are removed from the hot metal in the oxygen converter. A reduction process is one possibility to return these metals into the product cycle. The objective is to prevent the formation of a compound of iron and phosphorus to form iron phosphides as it causes an accumulation of phosphorus in the crude steel if reused internally. In a novel reactor concept, which has been developed at the Chair of Thermal Processing Technology at the Montanuniversitaet Leoben, the complete recovery of the metals with parallel removal of the phosphorus via the gas phase is possible. It is based on the inductive heat input via a graphite bed, which offers a particularly large surface area. In a series of preliminary experiments, the optimum mixture of BOF slag (BOFS) and slag formers as well as the optimum operating temperature were determined. As a result, the feed mixture could be successfully tested in continuous operation. The results show that the slag is almost completely free of iron, chromium, and phosphorus after treatment.

Keywords: Converter slag, Phosphorus recovery, Slag reduction, Metal recovery

\section{Einleitung}

Die weltweite Rohstahlerzeugung basiert zu zwei Drittel auf der Hochofen-Konverter-Route [1]. Bei der Herstellung von Rohstahl auf der Hochofen-Konverter-Route mittels Linz-Donawitz-Verfahrens (LD) entsteht ein mengenmäBig relevanter Massenstrom an Stahlwerksschlacke (LDSchlacke, LDS), von über $110 \mathrm{~kg} / \mathrm{t}$ Rohstahl [2]. Dies bedeutet in Österreich eine jährliche Menge an LD-Schlacke von etwa $800.000 t$, welche typischerweise Wertstoffe wie Eisen, Phosphor, Mangan und Chrom enthält [3]. Neben dem großen Sekundärrohstoff- und Wärmepotential stellt zusätzlich die aktuelle Gesetzeslage in Österreich bezüglich der Einsetzbarkeit im Straßenbau von LD-Konverterschlacken sehr fordernde Aufgaben an die Industrie und in weiterer Folge an die Wissenschaft. Der Umstand, dass 
nach der gültigen Recycling-Baustoffverordnung neben dem Eluatgehalt auch der Gesamtgehalt von Chrom für die Einsatztauglichkeit im Straßenbau entscheidend ist, führt aktuell dazu, dass die Schlacke nach mechanischer Aufbereitung und Abtrennung von kleinen Fraktionen magnetischer Bestandteile deponiert oder zwischengelagert werden muss [4]. Am Lehrstuhl für Thermoprozesstechnik (TPT) wird aktuell die langjährige Erfahrung in der Behandlung oxidischer Reststoffe auf die LD-Schlacke angewandt, und es werden diverse Möglichkeiten untersucht, das Potential der Schlacke zu nutzen. Die Ziele sind neben der Rückgewinnung von Wertstoffen wie Eisen, Mangan und Chrom auch die Verwertbarkeit der Restschlacke und die Nutzung des Wärmepotentials. Ein großes Hindernis bei der Rückgewinnung der Metalle ist die Anreicherung von Phosphor im Eisen bei hohen Temperaturen, welche nach aktuellem Stand der Technik die Abtrennung von Phosphorverbindungen aus der LDS erschwert. Dieses Problem bei einer klassischen Reduktion im Schmelzbad soll mit einem neuartigen Reaktorkonzept durch eine induktiv erwärmte Grafitschüttung gelöst werden. Zur Verwertbarkeit der nach der Reduktion entstehenden Restschlacke wird eine Schlackenqualität angestrebt, welche der Qualität von Hüttensand entspricht, sodass sich die Schlacke als Zuschlagsstoff für die Zementindustrie eignet. Zur Nutzung des Wärmepotentials wird eine Restschlackenzusammensetzung angepeilt, die es ermöglicht, die Schlacke mittels eines Rotationsverfahrens trocken zu granulieren.

\section{Forschungsarbeit}

Nachfolgend wird auf die angestellten Versuche und Reaktorkonzepte zur Zielerreichung eingegangen.

\subsection{Reduktion im induktiv beheizten kontinuier- lichen Prozess}

Die Untersuchung der Verwertung von LDS mit neuen Methoden in unterschiedlichen Stoßrichtungen steht im Rahmen des K1-MET-Projektes 1.2 "Utilization of metallurgical slags" im Mittelpunkt. Am TPT soll das Verhalten von LDS im am Lehrstuhl entwickelten InduRed-Reaktor untersucht werden. Der InduRed-Reaktor ist ein Schüttschichtreaktor aus Grafitelementen, die mittels Induktion direkt auf $1650^{\circ} \mathrm{C}$ erhitzt werden (Anm.: für andere potenzielle Anwendungen sind bis zu $2100^{\circ} \mathrm{C}$ erreichbar) [5]. Das Reaktorkonzept ist schematisch in Abb. 1 dargestellt.

Das Prinzip des Reaktors für einen kontinuierlichen Betrieb beruht auf drei wassergekühlten Induktionsspulen, welche die Grafitschüttung im Inneren eines Keramikaufbaus direkt erwärmen. Das Versuchsmaterial durchläuft auf seinem Weg durch den Reaktor mehrere Zonen, in welchen das Aufschmelzen, die Reduktion und der Austrag vonstattengehen. Das Material wird dabei feinkörnig im festen $\mathrm{Zu}$ stand eingebracht, die Austragsströme verteilen sich auf eine flüssige Schlackenphase, eine flüssige Metallphase und eine Gasphase, welche auf halber Reaktorhöhe abgezogen wird. Die Besonderheiten des Konzeptes sind:

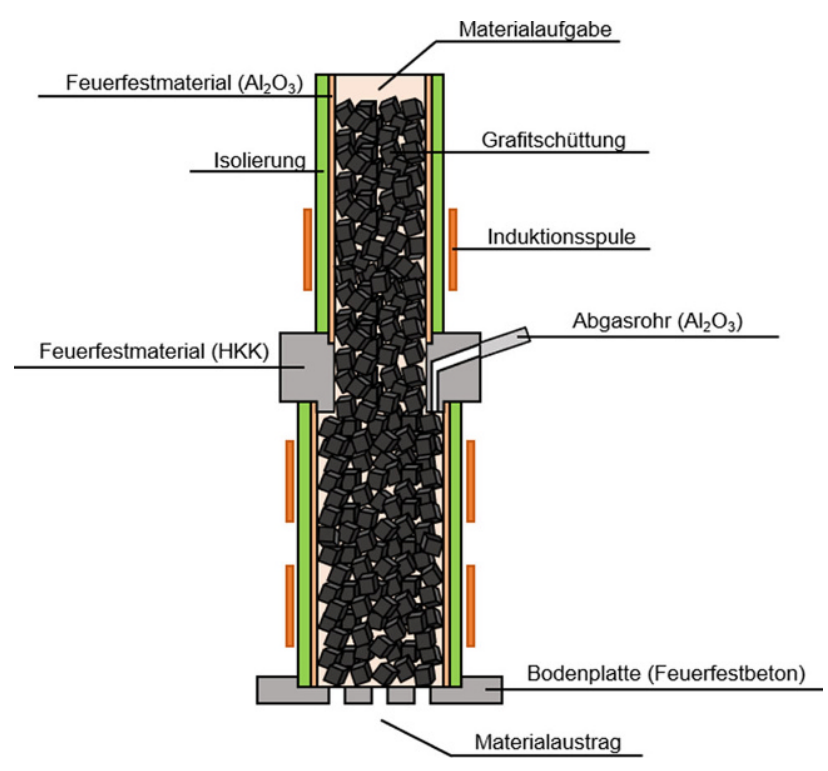

Abb. 1: Schematische Darstellung des InduRed-Reaktors [6]

- der sehr geringe Sauerstoffpartialdruck

- die Möglichkeit, entstehende Gase punktuell abziehen zu können und

- die Grafitschüttung, welche eine große Oberfläche bietet, sodass sich ein dünner Schmelzfilm ausbildet und Diffusions- und Desorptionsvorgänge von Phosphorverbindungen in Schlacke und Metall schnell ablaufen können

Die Kombination der beiden letztgenannten Punkte verhindert die Anreicherung des Phosphors im Eisen, was wiederum die Produktqualität enorm steigert und den hütteninternen Wiedereinsatz ermöglichen kann.

Um mit der LDS Versuche in der bereits bestehenden Pilotanlage durchführen zu können, war es notwendig, das Ausgangsmaterial hinsichtlich seiner Eigenschaften und der Eignung für den Prozess zu untersuchen. Die wichtigsten Punkte sind dabei die Viskosität bei bestimmten Temperaturen, die Reduzierbarkeit verschiedener Inhaltsstoffe wie Chrom und Phosphor und die Fließfähigkeit der resultierenden Restschlacke.

\subsection{Entwicklung der Vorversuchsanlage InduMelt}

Um Materialien hinsichtlich dieser Punkte untersuchen zu können, entstand am TPT eine Versuchsanlage mit dem Ziel, die herrschenden Bedingungen im Batchbetrieb mit kleineren Probemengen und geringerem Versuchsaufwand nachzustellen. Die in der Folge InduMelt genannte Anlage kann je nach Versuchsaufbau für unterschiedlichste Forschungsaufgaben herangezogen werden. Die Technik basiert auf den Induktionseinheiten der kontinuierlichen InduRed-Pilotanlage, jedoch beschränkt sich die InduMelt-Anlage auf eine wassergekühlte Spule und ist aufgrund des modularen Aufbaus standortunabhängig einsetzbar. Der Leistungseintrag kann entweder in Schüttungen, Ringe oder 


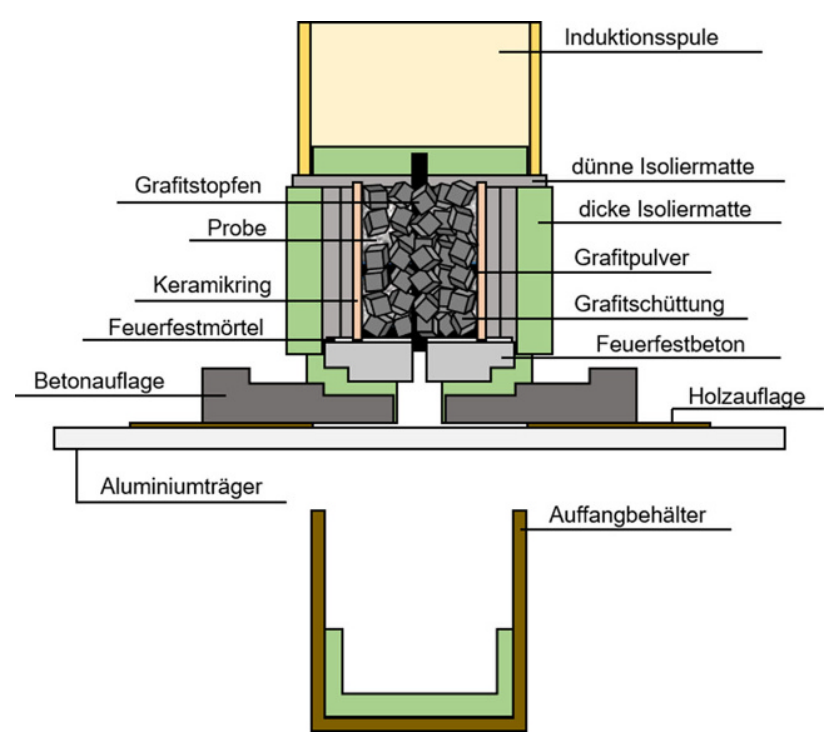

Abb. 2: Tiegelkonzept der Reduktionsversuche [6]

Tiegel aus leitendem Material erfolgen. Somit bieten sich sehr viele Möglichkeiten, neue Materialien bewerten und untersuchen zu können. Der ringförmige Aufbau wurde für veränderungsfreies Erstellen verschiedener Schlackenmischungen konzipiert, der Aufbau mit Schüttung dient zur reduzierenden Behandlung (Abb. 2).

Im Folgenden wird die Versuchsdurchführung zur Optimierung und Untersuchung der Schlackenzusammensetzung mit Additivbeimengung beschrieben.

\subsubsection{Optimierung der Fließfähigkeit und Überprüfung der Reduzierbarkeit}

In einem ersten Schritt wurde die Fließfähigkeit des Ausgangsmaterials optimiert, da diese entscheidend für die Behandlung im kontinuierlichen Betrieb ist. Um die LDSchlacke diesen Anforderungen entsprechend anzupassen, ist die Beimengung einer Siliziumquelle notwendig. Zur Erstellung dieser neuen Schlackenmatrix und Senkung der Basizität $\left(\mathrm{B}_{2}=\mathrm{CaO} / \mathrm{SiO}_{2}\right.$ in Massenprozent (m.$\%)$ ) wurde in zahlreichen Vorversuchen Quarzsand bzw. Hochofenschlacke der LD-Schlacke in unterschiedlichen Mengenverhältnissen zugegeben. Die Erstellung einer homogenen Mischung wurde dabei in einem separaten Schritt durchgeführt. Das Reaktorkonzept wurde dafür so modifiziert, dass der Wärmeeintrag über die induktive Erwärmung eines Grafitrings um den Keramikring erfolgt und es zu keinem Kontakt mit Kohlenstoff kommt. Dieser Ansatz begründet sich auf der Intention, dass es lediglich zu einer Homogenisierung und keiner Reduktion kommen soll. Der Aufbau ist in Abb. 3 ersichtlich.

Nach dem Schmelzen der beiden Komponenten in diesem Aufbau wurde die neue Schlacke über ein $8 \mathrm{~mm}$ großes Loch am unteren Ende des Reaktors abgestochen. Dabei wurde der Durchmesser des Schlackenstroms beobachtet. Von jeder Mischung wurden 2,5 kg geschmolzen und abgestochen. Sofern die Fließfähigkeit nach optischer Bewer-

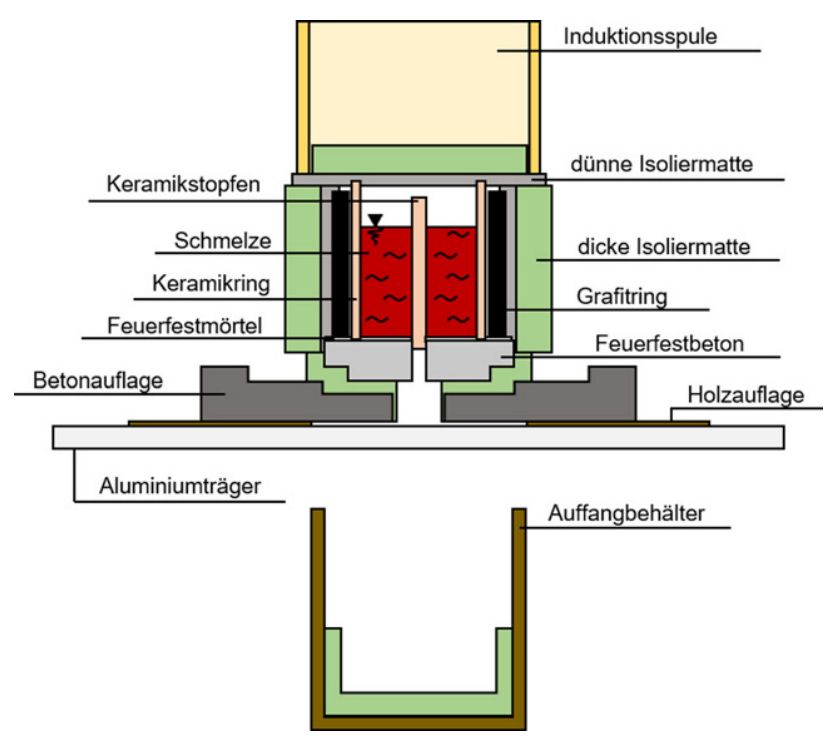

Abb. 3: Tiegelkonzept bei Schmelzversuchen [6]

tung zufriedenstellend war, wurde diese nach dem Erstarren bei Umgebungstemperatur auf $<1 \mathrm{~mm}$ gebrochen.

Im darauffolgenden Reduktionsschritt wurden davon $0,5 \mathrm{~kg}$ mit $50 \mathrm{~g}$ reaktivem Kohlenstoff gemischt und mit Grafitwürfeln in den Reaktoraufbau (Abb. 2) chargiert. Der Temperaturverlauf des Reduktionsprozesses wurde folglich nach den Reduktionstemperaturen der enthaltenen Metalloxide und Phosphorverbindungen gewählt. Ziel war es, eine mögliche selektive Reduktion der Oxide zu untersuchen. Es ist zu erwähnen, dass bei diesem Reduktionsvorgang die Reaktoratmosphäre nicht verändert und kein Abgas abgezogen wurde. In Abb. 4 ist die Versuchsdurchführung dargestellt.

\subsection{Kontinuierlicher Betrieb}

Aus den Vorversuchen konnte die optimale Schlackenzusammensetzung zur weiteren Behandlung definiert werden. In einem ersten Versuch konnten 2,5 kg dieses Gemisches aus LD-Schlacke und Quarzsand im kontinuierlichen Betrieb der InduRed-Pilotanlage untersucht werden. Dieses Anlagenkonzept zeichnet sich unter anderem durch die Möglichkeit eines kontinuierlichen Gasabzugs mit nachgeschalteter Verbrennung aus. Das Prozessgas, bestehend aus $\mathrm{CO}$ und $\mathrm{P}_{4}$, wird nach der Brennkammer als Phosphorpentoxid $\left(\mathrm{P}_{2} \mathrm{O}_{5}\right)$ in den Gaswäscher überführt und kann folglich als Phosphorsäure dem Gesamtprozess entnommen werden. Bei grafischer Gegenüberstellung der Probenaufgabemenge und der Brennkammertemperatur ist es möglich, die Produktion von $\mathrm{P}_{4}$ und $\mathrm{CO}$ sofort $\mathrm{zu}$ detektieren. Eine weitere Möglichkeit dafür bietet eine kontinuierliche Messung des $\mathrm{pH}$-Wertes des Prozesswassers des Wäschers. Darüber hinaus kann die Phosphorsäure zukünftig bedarfsabhängig in unterschiedlichen Konzentrationen abgezogen werden. Abb. 5 veranschaulicht die InduRed Pilotanlage am Lehrstuhl für Thermoprozesstechnik der Montanuniversität Leoben. 
Abb. 4: Überblick der Versuchsdurchführung [6]

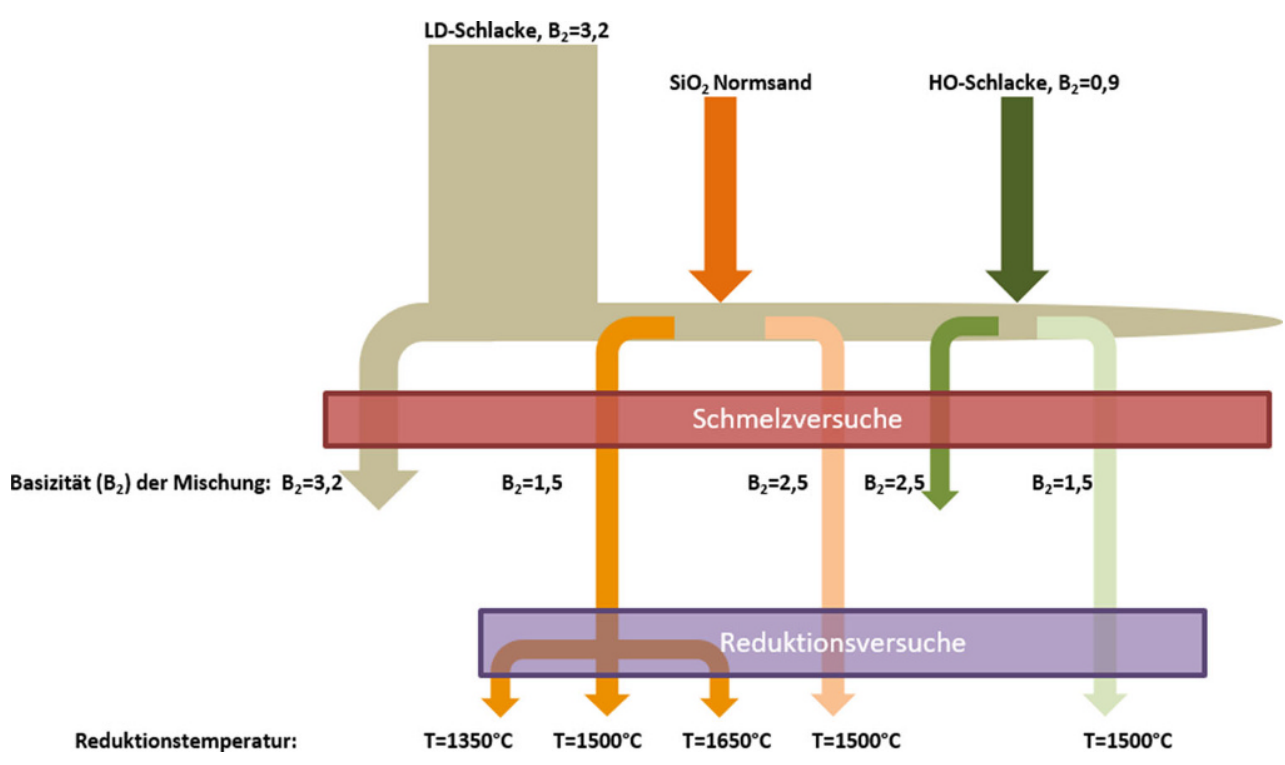

3. Ergebnisse

In diesem Abschnitt werden die Ergebnisse der durchgeführten Versuche dargestellt.

\subsection{Vorversuche}

Die Experimente zur Verbesserung der Fließfähigkeit und Untersuchung des Reduktionsverhaltens durch Modifizierung der LD-Schlacke und anschließenden Reduktion in der InduMelt-Anlage waren vielversprechend. Die besten Ergebnisse bezüglich der Reduktionsgrade einzelner Metalloxide konnten bei einer Mischung der LD-Schlacke mit Quarzsand bei einer Basizität von 1,5 und einer Prozesstemperatur von $1650^{\circ} \mathrm{C}$ erzielt werden. Nach Versuchsende konnten insgesamt drei Fraktionen detektiert und analysiert werden (Abb. 6).

- Metallkugeln als Abscheidung auf den Grafitwürfeln

- Abgestochenes Material im Auffangbehälter

- Restschlacke als Rückstand im Tiegel

In weiterer Folge wurden aus der Analyse die jeweiligen Reduktionsgrade mit folgender Gl. 1 ermittelt. Dies war not-

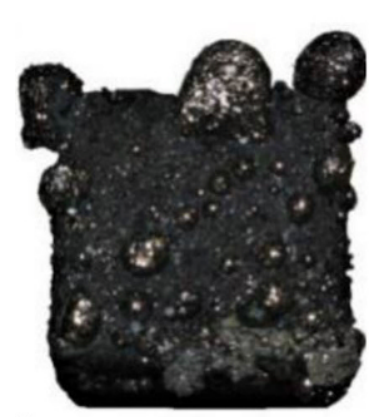

a

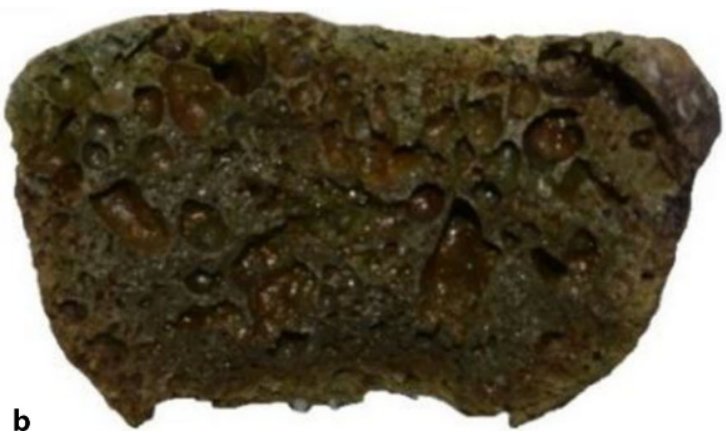

Abb. 6: Metallkugeln auf einem Grafitwürfel, Abstich und Restschlacke aus der Behandlung von LDS $+Q S$ mit B2 = 1,5 im Batch-Betrieb (v. I. n. $r$ ) [7] 


\begin{tabular}{|c|c|c|c|}
\hline \multicolumn{4}{|c|}{$\begin{array}{l}\text { TABELLE } 1 \\
\text { Reduktionsgrade der Vorversuche von LDS mit } \\
\text { Quarzsand bzW. Hochofenschlacke mit } B_{2}=1,5 \text { bei } \\
1500^{\circ} \mathrm{C} \text { bzw. } 1650^{\circ} \mathrm{C}[6]\end{array}$} \\
\hline Additiv & Quarzsa & & Hochofenschlacke \\
\hline Temperatur & $1500^{\circ} \mathrm{C}$ & $1650^{\circ} \mathrm{C}$ & $1650^{\circ} \mathrm{C}$ \\
\hline Eisen & $90,1 \%$ & $99,4 \%$ & $96,8 \%$ \\
\hline Phosphor & $90,2 \%$ & $99,2 \%$ & $91,9 \%$ \\
\hline Chrom & $74,1 \%$ & $95,6 \%$ & $80,7 \%$ \\
\hline Mangan & $14,0 \%$ & $46,5 \%$ & $17,2 \%$ \\
\hline
\end{tabular}

\begin{tabular}{|c|c|}
\hline \multicolumn{2}{|c|}{$\begin{array}{l}\text { TABELLE } 2 \\
\text { Phosphorverteilung auf die einzelnen Fraktionen } \\
\text { nach einem Vorversuch LDS }+0.5 \text { mit } B_{2}=1,5 \text { bei } \\
1650^{\circ} \mathrm{C}[6]\end{array}$} \\
\hline Fraktion & Phosphorgehalt (\%) \\
\hline Gas & 79,0 \\
\hline Metall & 15,6 \\
\hline Schlacke & 0,3 \\
\hline Restschlacke & 5,1 \\
\hline
\end{tabular}

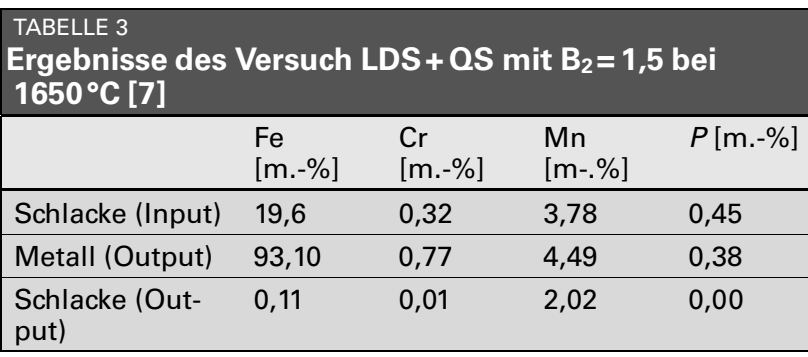

wendig, da aufgrund der fein verteilten Metallkugeln die Schließung einer Massenbilanz nicht möglich war.

\section{Reduktionsgrad}

$=\left(1-\frac{\text { Elementgehalt nach der Reduktion }[\%(w / w)]}{\text { Elementgehalt vor der Reduktion }[\%(w / w)]}\right)$ (1) $\cdot 100 \%$

Die Reduktionsgrade der Vorversuche sind Tab. 1 zu entnehmen.

Die flüchtigen Bestandteile wurden wiederum bei der genaueren Betrachtung des Phosphors, im Speziellen in der
Gasphase, miteinbezogen. Für die Metallfraktion ist eine gravimetrische Betrachtung aufgrund der zu geringen Menge in der Schlackenmatrix nicht möglich. Alternativ werden die in einer ICP-MS (Massenspektrometrie mit induktiv gekoppeltem Plasma) analysierten Elementgehalte in einer iterativen Berechnungsmethode verwendet, um die Zusammensetzung der Schlacke- und Metallfraktionen zu identifizieren. Die daraus resultierenden Ergebnisse werden folglich mit den theoretisch ermittelten Gehalten, basierend auf deren chemischen Zusammensetzung, verglichen. Eine gute Korrelation konnte festgestellt werden. Die Verteilung des Phosphors nach dem Versuch auf die einzelnen Fraktionen ist Tab. 2 zu entnehmen.

Die angeführte Restschlacke bezieht sich auf jene Schlacke, welche während der Probennahme zwischen den Grafitstücken auffindbar war. Es kann angenommen werden, dass auch diese ausreduziert abgestochen werden hätte können, sofern die Menge der Eingangsschlacke für ein ausreichendes Durchflussverhalten genügt hätte.

Bezugnehmend auf den erfolgreichsten Versuch (LDS + Quarzsand bei $1650^{\circ} \mathrm{C}$ ) konnten im Detail die Ergebnisse aus Tab. 3 erzielt werden.

Aus einer röntgendiffraktometrischen Untersuchung (RDA) kombiniert mit einer Analyse im Elektronenmikroskop (REM) geht hervor, dass die Schlacke nach der Reduktion frei von Eisen, Chrom und Phosphor ist. Die Entfernung von Mangan aus der Schlacke ist unter anderem aufgrund des Reduktionsverhaltens von Manganoxiden und der Präsenz von Schwefel, welcher mit Mangan eine Verbindung zu MnS eingeht, nicht vollständig möglich. Aus den REM-Aufnahmen (Abb. 7b) geht hervor, dass die resultierende Schlacke nach dem Reduktionsversuch eine nahezu homogene Schlacke ausbildet. Dies wiederum ist ein Indiz dafür, dass das spinellbildende Chrom (Abb. 7a) aus der Struktur weitestgehend entfernt werden konnte, was wiederum den Ergebnissen aus der ICP-MS entspricht.

\subsection{Kontinuierlicher Betrieb}

Die Ergebnisse aus den Vorversuchen konnten auch im kontinuierlichen Betrieb reproduziert werden. Die Extraktion des Phosphors wurde über den Anstieg der Brennkammertemperatur, wie auch durch $\mathrm{pH}$-Wertmessung des Prozesswassers, festgestellt. Daraus war ersichtlich, dass die Phosphorentfernungsrate zwischen 65 und $80 \%$ liegt. Analysen
Abb. 7: REM-Aufnahme der Schlacke aus dem Vorversuch $\mathrm{LDS}+\mathrm{QS}$ mit $\mathrm{B}_{2}=1,5$ nach dem Schmelzvorgang (a) und dem Reduktionsvorgang bei $1650^{\circ} \mathrm{C}$ (b) [6]
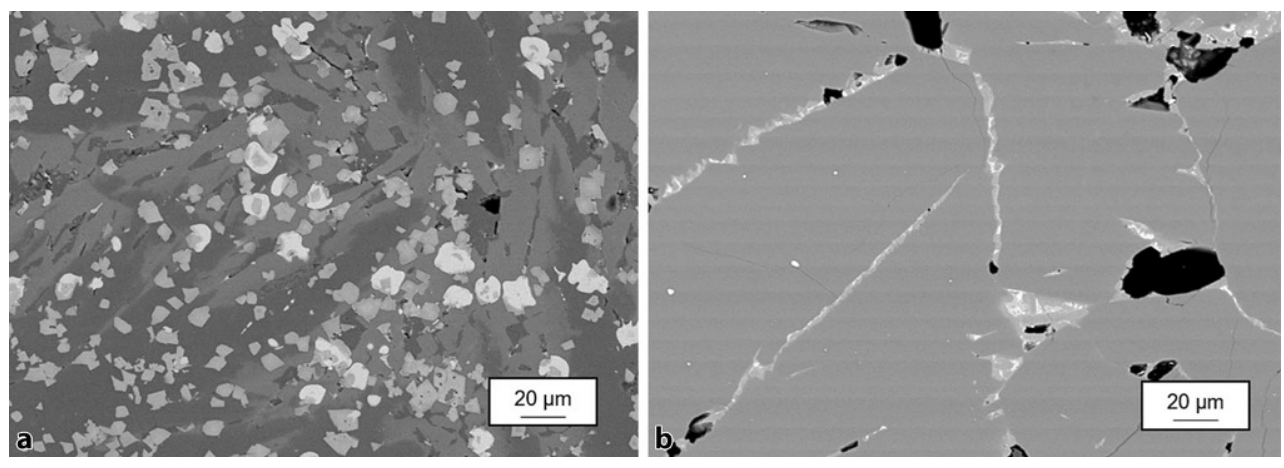
ergaben, dass die produzierte Schlacke frei von Chrom, Eisen und Phosphor war und derselbe Gehalt an MnS wie in den Vorversuchen erreicht wurde. Bei Betrachtung der Metallfraktion ist erkennbar, dass die Qualität des Eisens aufgrund der Bildung kleiner über den Reaktorquerschnitt verteilter Kugeln nicht einheitlich ist. Der Phosphorgehalt variiert dabei erheblich, weshalb weitere Analysen angestellt werden müssen, um die Phosphorverteilung beurteilen zu können.

\section{Zusammenfassung und Ausblick}

Das neuartige Reaktorkonzept ermöglicht die Gewinnung einer Schlacke, welche nahezu vollständig frei von Eisen, Chrom und Phosphor ist. Aus den Vorversuchen geht hervor, dass der Chromgehalt in der mineralischen Fraktion lediglich 0,01 m.-\% beträgt, wodurch die Bestimmungen der Recycling-Baustoffverordnung erfüllt werden und die Ergebnisse einen Einsatz im Straßenbau erlauben würden. Die entstehende Metallfraktion besteht aus über 93 m.-\% Eisen und lediglich 0,4 m.-\% Phosphor. Dadurch wäre eine Wieder- bzw. Weiterverwendung im Hüttenwerk unter gewissen Bedingungen vorstellbar. Bis nahezu $85 \%$ des Phosphors konnten über die Gasphase aus dem Material entfernt werden. Ähnlich hohe Ergebnisse konnten auch im kontinuierlichen Betrieb realisiert werden. Zwischen 65 und $80 \%$ des Phosphors aus der eingesetzten LD-Schlacke konnten entfernt werden. Eisen-, Chrom- und Manganoxid konnten vollständig reduziert werden, lediglich Mangan verblieb in der Schlacke als MnS.

Der Fokus der Forschung wird sich zukünftig auf die Produktqualität der Schlacken- und Metallfraktionen richten. Durch Modifikation des Einsatzmaterials soll eine Schlacke produziert werden, welche den Anforderungen der Zementindustrie entspricht. Im Detail sollen Experimente zur Herstellung von latent-hydraulischen Phasen in reduzierter LDSchlacke mit simultaner Phosphorentfernung durchgeführt werden. Die Qualität der Metallfraktion soll durch konstruktive Änderungen am Reaktor sowie durch eine alternative Verfahrensroute (weiterer Frischprozess und selektive Anreicherung von Chrom und Phosphor in der zu behandelnden Schlacke) weiter verbessert werden.

Danksagung. Die vorliegende Arbeit wird im Rahmen des österreichischen Kompetenzzentren-Programms COMET K1-MET mit Mitteln des BMVIT, BMDW der Länder Oberösterreich, Steiermark und Tirol gefördert und zusätzlich von den Industriepartnern SCHOLZ Austria GmbH, Primetals Technologies Austria
$\mathrm{GmbH}$, voestalpine Stahl GmbH und voestalpine Stahl Donawitz GmbH mitfinanziert.

Funding. Open access funding provided by Montanuniversität Leoben.

Open Access Dieser Artikel wird unter der Creative Commons Namens nennung 4.0 International Lizenz veröffentlicht, welche die Nutzung, Vervielfältigung, Bearbeitung, Verbreitung und Wiedergabe in jeglichem Medium und Format erlaubt, sofern Sie den/die ursprünglichen Autor(en) und die Quelle ordnungsgemäß nennen, einen Link zur Creative Commons Lizenz beifügen und angeben, ob Änderungen vorgenommen wurden.

Die in diesem Artikel enthaltenen Bilder und sonstiges Drittmaterial unterliegen ebenfalls der genannten Creative Commons Lizenz, sofern sich aus der Abbildungslegende nichts anderes ergibt. Sofern das betreffende Material nicht unter der genannten Creative Commons Lizenz steht und die betreffende Handlung nicht nach gesetzlichen Vorschriften erlaubt ist, ist für die oben aufgeführten Weiterverwendungen des Materials die Einwilligung des jeweiligen Rechteinhabers einzuholen.

Weitere Details zur Lizenz entnehmen Sie bitte der Lizenzinformation auf http://creativecommons.org/licenses/by/4.0/deed.de.

\section{Literatur}

1. World Steel Association, World Steel in Figures, https://www. worldsteel.org/en/dam/jcr:f9359dff-9546-4d6b-bed0-996201185b12/ World\%2520Steel\%2520in\%2520Figures\%25202018.pdf (16. Dezember 2019).

2. Doschek, K.; Raupenstrauch, H.; Krenn, J.; Rauter, M.; Mally, V.; Geier, B.: Sekundärrohstoff- und Wärmerückgewinnung aus Eisenhüttenschlacken, in Schlackensymposium, 2016, S. 75-87

3. voestalpine, LD-Schlacke Zahlen und Fakten, https://www. voestalpine.com/group/static/sites/group/.downloads/de/konzern/ 2013-weissbuch-Id-schlacke.pdf (05. Dezember 2019)

4. Bundesminister für Land- und Forstwirtschaft, Umwelt und Wasserwirtschaft, Recycling-Baustoffverordnung RBV, 28.10.2016, https:// www.ris.bka.gv.at/q21 (05. Dezember 2019)

5. Schönberg, A.; Samiei, K.; Kern, H.; Raupenstrauch, H.: Der RecoPhos-Prozess - Rückgewinnung von Phosphor aus Klärschlammasche, Österr Wasser- und Abfallw, 66 (2014), Nr. 11-12, S. 403-407

6. Ponak, C.; Windisch, S.; Mally, V.; Raupenstrauch, H.: Recovery of Manganese, Chromium, Iron and Phosphorus from Basic Oxygen Furnace Slags, in GDMB (Ed.): European Metallurgical Conference 2019, Optimum utilization of resources and recycling for a sustainable solution, vol. 3., Clausthal-Zellerfeld: GDMB Verlag $\mathrm{GmbH}$, 2019, S. $1311-1319$

7. Windisch, S.; Ponak, C.; Mally, V.; Raupenstrauch, H.: Untersuchung der Möglichkeiten einer reduzierenden Behandlung von LD-Schlacke in einem induktiv beheizten Koksbett, in Schlackensymposium, 2018.

Hinweis des Verlags. Der Verlag bleibt in Hinblick auf geografische Zuordnungen und Gebietsbezeichnungen in veröffentlichten Karten und Institutsadressen neutral. 